\title{
La ISO 9001 y la administración de la calidad total en las empresas peruanas
}

\section{ISO 9001 and Management of Total Quality in Peruvian Companies}

\section{A ISO 9001 e a administração da qualidade total nas empresas peruanas}

\author{
Jorge Benzaquen de las Casas
}

\author{
Fecha de recibido: 5 de septiembre de 2017 \\ Fecha de aprobado: 30 de enero de 2018 \\ Doi: http://dx.doi.org/10.12804/revistas.urosario.edu.co/empresa/a.6056
}

Para citar: Benzaquen de las Casas, J. (2018) La ISO 9001 y la Administración de la Calidad Total en las Empresas Peruanas. Universidad \& Empresa, 20(35), 281-312. Doi: http://dx.doi.org/10.12804/revistas. urosario.edu.co/empresa/a.6056

\section{RESUMEN}

El presente estudio muestra el impacto de tener un Sistema de Gestión de Calidad (SGC) con la certificación ISO 9001 en las empresas en Perú con base en los nueve factores de éxito utilizados para medir la implementación de la Administración de la Calidad Total (TQM). En 2017 se analizaron 211 empresas, unas con dicha certificación y otras sin esta, las cuales fueron comparadas entre sí. Asimismo, se propone que la metodología utilizada en este estudio se aplique en otros países latinoamericanos.

Palabras clave: Administración de la calidad total, TQM, calidad, ISO 9001, empresas en Perú.

Profesor de CENTRUM Católica Graduate Business School - Pontificia Universidad Católica del Perú. Correo electrónico: jbenzaq@pucp.pe 


\section{ABSTRACT}

This study shows the impact of having a quality management system with Iso 9001:2008 certifications in companies in Peru on the basis of the nine factors that measure the success of the total quality management (TQM) implementation. In 2017, 211 companies were analyzed with the aim of comparing Iso 9001 certified companies and those without the certification. This study also proposes that the methodology used for this study might be applied in other Latin American countries.

Keywords: TQM, ISO 9001, quality, companies in Peru.

\section{RESUMO}

O presente estudo mostra o impacto de ter um Sistema de Gestão de Qualidade (SGC) com a certificação ISO 9001 nas empresas no Peru em base aos nove fatores de sucesso utilizados para medir a implementação da Administração da Qualidade Total (TQM). No ano 2017 se analisaram 211 empresas ao realizar uma comparação entre as empresas que possuem dita certificação e aquelas que não a têm. Igualmente, se propõe que a metodologia utilizada neste estudo se aplique em outro países latino-americanos.

Palavras-chave: Administração da qualidade total, TQM, qualidade, ISO 9001, empresas no Peru. 


\section{INTRODUCCIÓN}

La gestión de la calidad es una herramienta de suma importancia a nivel organizacional, por ello, muchas empresas deciden implementar el estándar y obtener la certificación ISO 9001. El objetivo de este artículo de investigación es comparar el desempeño de las empresas peruanas que cuentan con esta certificación y aquellas que no la tienen, con la finalidad de analizar cómo impacta este hecho en los factores de éxito de la Administración de la Calidad Total (TQM, por sus siglas en inglés).

La presente investigación es una continuación del estudio que se realizó en 2011 en Perú (Benzaquen, 2013) y toma como referencia la metodología utilizada por el autor en Colombia y Ecuador (Benzaquen, 2015; 2016), en donde en ambos casos se evidenció que las empresas que cuentan con el certificado ISO 9001 tienen un mejor promedio en los factores de TQM. Este artículo describirá el nivel de calidad en las empresas peruanas con respecto a los nueve factores de TQM estudiados en 2017.

\section{REVISIÓN DE LA LITERATURA}

\subsection{Calidad}

El concepto de calidad ha ido evolucionado con el paso del tiempo. La humanidad ha estado estrechamente vinculada con la calidad desde los tiempos en que el hombre comenzó a elaborar sus productos, es decir, alimentos, ropas, armas, entre otros (Velázquez, Terraza \& Ruiz, 2014).

La Organización Internacional de Normalización (ISO 9000) define calidad como "el grado en que un conjunto de características inherentes cumple con los requisitos" (ISO 9000,2005 , p.8), siendo estos requisitos tanto implícitos como explícitos. De la misma manera, esta organización definió el concepto de requisito como la "necesidad o expectativa establecida, generalmente implícita u obligatoria" (ISO 9000, 2005, p.8). Bajo este enfoque, se debe entender que la calidad está definida por el cliente, a través de lo que establece como necesidad o expectativa sobre un bien o servicio. 
Por otro lado, la Sociedad Americana para la Calidad (American Society for Quality, ASQ) establece dos significados básicos. El primero concibe la calidad como las características que posee un bien o servicio y que le dan la capacidad de satisfacer las necesidades de los clientes, ya sean explícitas o implícitas; el segundo expresa que la calidad es un bien o servicio que no tiene deficiencias (ASQ, s.f.).

El término "calidad" se asocia a la excelencia en el producto final, el cual satisface y supera las expectativas del cliente. Estas últimas están enmarcadas en el beneficio de utilizar el producto adquirido versus el precio pagado. Además, se precisa que, de acuerdo con la norma A31987 ANSI/ASQC, "calidad es la totalidad de aspectos y características de un producto que permiten satisfacer necesidades implícitas o explícitamente formuladas" (ASQ, s.f., [en línea]). Con ello se entiende que la calidad tiene relación con las expectativas del cliente respecto al bien o servicio por adquirir, tal como lo indica el ISO 9001.

Una manera bastante simple de entender la calidad deriva de las letras que componen este término formando un decálogo de calidad (figura 1). Así, tenemos la letra "C" de cliente, que indica que se tiene que satisfacer y exceder sus requerimientos, considerando sus necesidades y expectativas. La "A" de aprender, porque es importante prepararse para cambiar y ser el impulsor de la nueva filosofía en la empresa, a fin de mejorar la calidad; es necesario aprender mediante la capacitación para hacer los trabajos de manera correcta, porque las personas tienen que saber exactamente lo que deben hacer. La " $L$ " es de liderazgo, porque el involucramiento del líder en el proceso de mejora continua es esencial para poder alcanzar la excelencia en la empresa; el gerente general desempeña ese papel, sueña, ejecuta, ayuda y supervisa. La "I" es de innovación, porque para innovar tenemos que ser creativos y generar una idea, producirla y venderla; además, tenemos que investigar con el fin de desarrollar nuevos productos y proyectar un futuro sostenible. Es necesario incluir a todo el personal en el proyecto de cambio, ya que la calidad no es tarea de una persona o un grupo, sino de toda la empresa. La "D" es de diseño, porque nos hace pensar en lo importante que es traducir de manera adecuada los requerimientos del cliente en el lenguaje de la empresa, es decir, determinar los requisitos del producto y del proceso. La "A" es de adecuarse al uso del cliente con las especificaciones, la seguridad y el servicio 
postventa que éste necesita. Por último, la segunda " $D$ " es de disponibilidad porque nos hace pensar inmediatamente en la confiabilidad y mantenibilidad. Ambas características son inherentes al producto y reflejan el tiempo que podemos utilizarlo sin tener problemas; en el caso de que estos llegaran a producirse, la disponibilidad es el tiempo que va a pasar hasta que se restituya el estado operativo (Benzaquen, 2014). Este conjunto de normas ayudará a tener presente los aspectos más importantes que debemos considerar en las organizaciones.

Figura 1. Decálogo de la Calidad

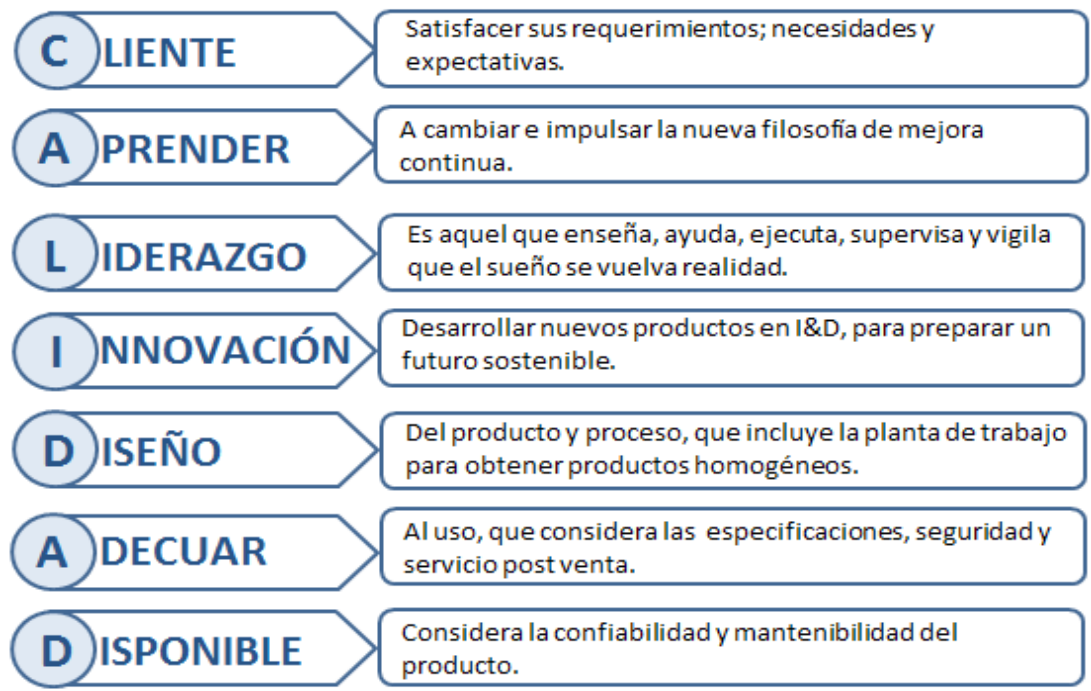

Fuente: elaboración propia.

\subsection{Administración de la Calidad Total (TQM)}

Las primeras nociones de calidad se caracterizaron por el enfoque en el producto, en el cumplimiento de las especificaciones y en la inspección para discriminar lo bueno de lo defectuoso. Pronto se pasó a la noción de control de calidad donde el enfoque se situó en los procesos; posteriormente, se amplió la noción para enfocarse en los sistemas mediante la perspectiva del aseguramiento de la calidad (Miranda, 2002).

Así, Huq y Stolen (1998) mencionaron que el TQM es una filosofía basada en el trabajo de Deming, 
Juran y Crosby. Ellos propusieron una aproximación estructurada que integra, facilita y enriquece el impacto de utilizar herramientas de análisis y control. Antony, Leung y Knowles (2002) realizaron una investigación empírica que demostró que si una organización adopta de manera efectiva el TQM, se puede tener beneficios como fortalecer el desempeño de la organización y mejorar su ventaja competitiva.

La TQM es un sistema basado en el enfoque total de sistemas que permiten a una organización desarrollar una cultura de mejoramiento continuo para cumplir su misión (Cantú, 2011). El modelo de TQM se puede definir como una filosofía de gestión cuyo objetivo es suministrar productos con un nivel de calidad que satisfaga a los clientes y que, al mismo tiempo, logre la motivación y satisfacción de los empleados, gracias a una mejora continua en los procesos de la organización y a la participación de todas las personas que forman parte de ella o que se relacionan con la misma (Miranda \& Chamorro, 2007).

Los objetivos de la TQM son: a) la satisfacción del cliente, b) la mejora de la calidad, c) la satisfacción del trabajador, d) el incremento de la competitividad y e) la satisfacción de la sociedad. Asimismo, esta herramienta tiene la necesidad de implementar la calidad en todos los niveles de la organización y de utilizar los siguientes principios fundamentales del sistema: a) consecución de la plena satisfacción de las necesidades y expectativas del cliente; b) desarrollo de un proceso de mejora continua en todas las actividades y procesos; c) total compromiso de la dirección y un liderazgo activo directivo; d) participación de todos los miembros de la organización y fomento del trabajo en equipo; e) involucración del proveedor en el sistema de la calidad total de la empresa; f) identificación y gestión de los procesos clave de la organización; g) toma de decisiones de gestión basada en datos y hechos; h) orientación al cliente, a la satisfacción de sus necesidades y expectativas; i) liderazgo de la dirección; j) participación de todos los empleados y la mejora continua (Sánchez, 2005).

Por otro lado, en un estudio más reciente, Androniceanu (2017) propuso un enfoque tridimensional de la TQM como una opción estratégica para lograr la excelencia en los negocios. Este enfoque indica que la TQM es un instrumento y una filosofía de gestión, por consiguiente, debe abordarse en tres dimensiones: técnica, social y económica. A su vez, estas dimensiones se agrupan 
en tres subsistemas. El subsistema técnico incluye los elementos de calidad, estándares y características técnicas; el subsistema económico, los elementos de calidad, parámetros del bien y/o servicio y costos, y el subsistema social, los elementos de calidad, precio y términos. Según Androniceanu (2017), con la implementación profesional de los parámetros antes mencionados se puede lograr la excelencia empresarial.

\subsection{Norma ISO 9001}

La Norma de la Organización Internacional de Normalización ISO 9001 especifica los requisitos para los Sistemas de Gestión de la Calidad (SGC) aplicables a todas las organizaciones que necesiten demostrar su capacidad para brindar productos que cumplan los requisitos de sus clientes y las reglas que correspondan. Esta norma tiene como objetivo aumentar la satisfacción del cliente y es aplicable en cualquier organización, sin importar el tipo o tamaño de la empresa, debido a que es aceptada a nivel mundial por el sector industrial y de manufactura (ISO, 2015).

La ISO 9001 estableció que "todos los requisitos de esta Norma Internacional son genéricos y se pretende que sean aplicables a todas las organizaciones sin importar su tipo, tamaño y producto suministrado" (ISO 9001, 2008, p.1). Esto significa que este sistema puede ser implementado por un supermercado, una empresa dedicada a la fabricación de tela, una institución dedicada a la educación o incluso un organismo del estado. Además, se estableció la adopción de un enfoque basado en procesos cuando se desarrolla, implementa y mejora la eficacia de un sistema de gestión de la calidad, para aumentar la satisfacción del cliente mediante el cumplimiento de sus requisitos (ISO 9001, 2008, p.6).

A través de los años, esta norma ha tenido una fuerte penetración en los sectores de tecnología y prestación de servicios debido a los cambios suscitados en el mundo. Asimismo, el sector público puede mejorar sus índices de gestión, transparencia y buen gobierno mediante la implementación adecuada de modelos de gestión de calidad basados en el estándar ISO 9001 (Báez, 2013).

Con el tiempo, la ISO 9001 ha desarrollado cinco versiones: a) la primera versión, ISO 9001:87 (1987);

b) la segunda versión, ISO 9001:94 (1994); c) la tercera versión, ISO 9001:2000; d) la cuarta versión, ISO 9001:2008; y e) la quinta versión, ISO 9001:2015, usada en la actualidad. La norma ISO 9001 señala que 
la Dirección General debe ser la que asegure que los diversos directores de los departamentos se aproximen a un sistema de gestión, porque su evaluación $\mathrm{y}$ proceso de certificación aseguran los objetivos del negocio, favoreciendo además las mejoras prácticas de los trabajadores y procesos. Se especifica también lo que se necesita hacer para que un sistema de gestión de la calidad sea óptimo y se pueda usar a nivel interno organizacional para obtener certificaciones o para fines contractuales (Indecopi, 2010).

El principal aporte de la certificación de los sistemas de gestión ISO 9001 a la competitividad del país es el fortalecimiento de la infraestructura sectorial. Por lo tanto, se promueven las condiciones para el desarrollo científico y tecnológico de una nación, lo que significa que la certificación ISO 9001 le sirve a las organizaciones para competir dentro de un sector productivo (Bohórquez, 2010).

La norma ISO 9001:2015 se enfoca en los procesos e incorpora el ciclo Planificar, Hacer, Verificar y Actuar (PHVA), así como el pensamiento basado en riesgos. El enfoque en procesos desarrolla, implementa y mejora la eficacia para aumentar la satisfacción del cliente mediante el cumplimiento de los requisitos; además, permite las interrelaciones e interdependencias entre procesos, logrando así el desempeño global de la organización. La gestión de los procesos y el sistema en conjunto pueden ser posibles con el ciclo PHVA, el cual asegura que no falten los recursos en los procesos y que se gestionen adecuadamente, así como determina las oportunidades de mejora. Por otro lado, el pensamiento basado en riesgos se encarga de controles preventivos para minimizar los efectos negativos y maximizar el uso de las oportunidades a medida que se determinen aquellos factores que afectarían el cumplimiento de los resultados planificados (ISO, 2015).

\subsection{Relación entre la certificación ISO 9000 y la implementación de la TQM}

La norma ISO 9000 y la TQM son unos de los enfoques de calidad más utilizados en los últimos tiempos dentro de las empresas para cumplir los objetivos organizacionales. Por ejemplo, en los estudios enfocados en empresas en el Perú se halló la existencia de una relación entre los factores tomados para medir la TQM con los principios de la norma ISO 9000:2005. Benzaquen (2013) realizó una investigación en 2011 donde evaluó nueve factores de la TQM, evidenciándose una influencia 
positiva en las empresas con certificación ISO 9001 a comparación de las que no contaban con dicho certificado. De los nueve factores, los que tuvieron mayor promedio fueron la Alta Gerencia y el Planeamiento de la calidad.

Por otro lado, en una muestra con empresas de manufactura en Malasia, un estudio demostró que, en general, el enfoque basado en la norma ISO 9000 impulsa la adopción de prácticas de TQM. Sus resultados revelaron que la ISO 9000 y la TQM pueden coexistir e implementarse juntas en una sola organización y, por lo tanto, están interrelacionadas y no provocan conflictos en las organizaciones de producción (AlKhalili \& Subari, 2014).

Benzaquen y Convers (2015) realizaron otra investigación en las empresas de Colombia. El estudio halló que, de los nueve factores de la TQM evaluados en una muestra de 207 empresas, se obtuvo diferencias significativas en los nueve factores de TQM respecto a las empresas que no están certificadas. Los factores con mayor promedio fueron Liderazgo y Enfoque hacia la satisfacción del cliente.

En Estados Unidos, Aba, Badar y Hayden (2016) realizaron un estudio con una muestra de 397 empresas que habían recibido la certificación ISO 9001 desde 1991 hasta 2002; se incluyeron empresas certificadas y empresas no certificadas. El estudio concluyó que hubo una mejora significativa en el rendimiento del año anterior al año de certificación. Las empresas certificadas tuvieron un mejor rendimiento que las empresas no certificadas. El estudio concluyó que la presencia de un Sistema de Administración de la Calidad Total en las empresas certificadas podría ser responsable del mayor rendimiento operativo de estas durante un período de cinco años.

Benzaquen y Pérez-Cepeda (2016) realizaron una investigación en las empresas de Ecuador para analizar el impacto de los nueve factores de la TQM evaluados en una muestra de 211 empresas. Los resultados confirmaron que las compañías con certificación ISO 9001 tienen un mejor puntaje en los nueve factores de TQM analizados en el estudio, al ser comparadas con aquellas empresas que no están certificadas. De los nueve factores, los que obtuvieron un mayor promedio en las empresas con certificación fueron Planeamiento de la calidad y Liderazgo.

Finalmente, uno de los estudios más recientes en el área de la TQM es el de Miroslav, Slobodan, Vladimir y Tijana (2017), quienes realizaron 
una investigación acerca de la implementación de la TQM y la satisfacción de los clientes en el sector hotelero en Rumania. Se midieron las expectativas y la satisfacción de los clientes que se hospedaron en hoteles que habían implementado la TQM y que tenían la certificación ISO 9001, en comparación con aquellos hoteles que no estaban certificados ni tampoco habían implementado la TQM. Los resultados indicaron que los clientes que se hospedaron en los hoteles en donde se había implementado la TQM estaban más satisfechos con el servicio brindado.

\section{CALIDAD EN EL PERÚ}

Una de las primeras instituciones interesadas en cumplir las normas de calidad internacional fue la Sociedad Nacional de Industrias (SNI), fundada en 1896 con la finalidad de instaurar un organismo que represente al sector industrial y proteja los intereses de la industria privada nacional. La SNI se ha dedicado a fomentar la internacionalización de los productos peruanos cumpliendo los estándares de calidad internacional que marcan la tendencia de consumo a nivel global (SNI, s.f.).

El Centro de Desarrollo Industrial (CDI) forma parte de la SIN y brinda servicios de capacitación y asistencia técnica a empresas; también está a cargo del Encuentro Anual de Calidad en Perú, del Premio Nacional a la Calidad y da un reconocimiento a las organizaciones que certifican o recertifican su sistema de gestión (CDI, s.f.). En 1989, el CDI promovió la creación del Comité de Gestión de la Calidad (CGC). En un principio estuvo integrado por cuatro instituciones, pero hoy en día ascienden a 21. Estas organizaciones, a través de un acuerdo de cooperación, buscan promover la calidad en el Perú. Los integrantes más importantes son: a) la Asociación de Exportadores (Adex), b) la Cámara Peruana de la Construcción (Capeco), c) el Instituto Nacional de Defensa de la Competencia y de la Protección de la Propiedad Intelectual (Indecopi), d) el Ministerio de la Producción, e) la Pontificia Universidad Católica del Perú, entre otros (CDI, 2011).

En la década de 1990, las empresas peruanas tuvieron que afrontar una realidad distinta. A nivel internacional surgieron nuevas tendencias, como la globalización, las innovaciones tecnológicas, la apertura de los mercados y la privatización de las empresas estatales; finalmente, el país sufrió una inflación controlada que conllevó a una integración regional. Estas son solo algunas de las 
características que han marcado un cambio profundo en el entorno en el que operan las empresas y en el comportamiento de los consumidores (Mundaca et al., 2003).

En 2013, el Poder Ejecutivo presentó al Congreso de la República del Perú el Proyecto de Ley N³667/2013 PE, donde se planteaba la creación del Sistema Nacional para la Calidad (SNC) y el Instituto Nacional de Calidad (INACAL). Para 2014, el proyecto se aprobó a través del Decreto Supremo $\mathrm{N}^{\circ} 046-2014-\mathrm{PCM}$, publicado el 1 de julio de 2014. Mediante este decreto se aprobó la Política Nacional para la Calidad (Sociedad Nacional de Industria, 2014). El objetivo de esta iniciativa es promover la participación de entidades públicas y privadas que integran la Infraestructura Nacional de la Calidad conformada por las actividades de: a) normalización, b) acreditación, c) metrología y d) evaluación de la conformidad, que se realizan en el país (Gestión, 2014). Este sistema esta conformado por: a) el Consejo Nacional para la Calidad (CONACAL), b) el Instituto Nacional de la Calidad (INACAL) y sus comités técnicos permanentes y c) las entidades públicas y privadas que formen parte de la infraestructura de la calidad (Instituto Nacional de Calidad, 2014).

Según la base de datos de la Organización Internacional de Normalización (2017), en el Perú prevalece el número de empresas certificadas con la norma ISO 9001 en la versión del año 2008 desde 1993 hasta 2015. Aún son pocas empresas (3) las que están certificadas con la versión más reciente de ISO 9001 del año 2015, tal como podemos apreciar en la figura 2.

Figura 2. Certificación de empresas peruanas de 1993 a 2015

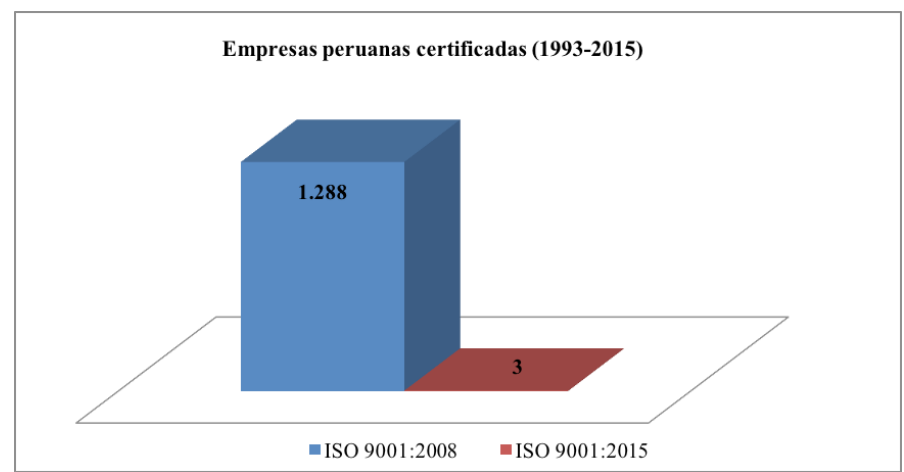

Fuente: Survey, 2017. 
Si bien se han hecho diversos esfuerzos en materia de gestión de calidad para asegurar una alta competitividad en la industria, el Instituto Nacional de Calidad afirmó durante el Foro Nacional "Cruzada por la Vida y la Seguridad de los Productos y Servicios" que "se tiene un total de 1.329 empresas con certificación de calidad ISO (ISO 9001 e ISO 14001), de un total de empresas formales activas en el Perú que llega a 1.382,899" ("Solo el 1\% de empresas," 2016, para. 2), según cifras de la SUNAT. Esto vendría a representar solo un $1 \%$ de empresas formales con sistemas de gestión de calidad implementados. Asimismo, se enfatizó que el Instituto Nacional de Calidad, como organización rectora del sistema para la calidad y ejecutor de la política nacional para la calidad, tiene el objetivo de promover la cultura de calidad para que el público solicite productos de calidad y así obligar a las empresas a implementar estos sistemas de gestión (INACAL, 2016).

\section{METODOLOGÍA}

Esta investigación analiza el impacto de la certificación ISO 9001 en los nueve factores de la TQM de las empresas certificadas y aquellas empresas que no poseen la certificación. Los factores de TQM analizados son los siguientes: a) LiderazgoAlta Gerencia; b) Planeamiento de la calidad; c) Control y mejoramiento del proceso; d) Auditoría y evaluación de la calidad; e) Gestión de la calidad del proveedor; f) Educación y entrenamiento; (g) Enfoque hacia la satisfacción del cliente; (h) Círculos de la calidad e i) Diseño del producto, de acuerdo al instrumento detallado del modelo utilizado en 2013 en Colombia (Benzaquen \& Convers, 2015).

En este estudio se quiere identificar si las empresas peruanas con certificación ISO 9001 tienen diferencias significativas al medir los nueve factores del TQM y al ser comparadas con aquellas que no están certificadas hasta 2017. Para lograr esto se realizó un cuestionario de 35 preguntas para poder validar si existe un impacto positivo en el nivel de calidad de las empresas peruanas que cuentan con la certificación ISO 9001.

En la figura 3 se muestran los factores agrupados en los cuatro bloques principales de la organización. 
Figura 3. Modelo de Nueve Factores de TQM en la Empresa.

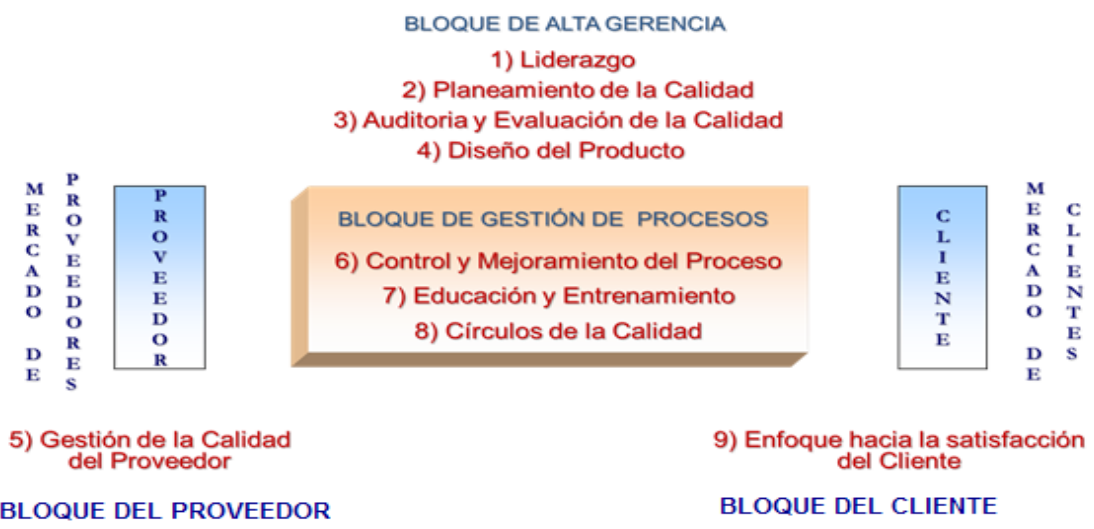

Fuente: Benzaquen y Convers, 2014.

\section{POBLACIÓN Y MUESTRA}

Este estudio consideró una población de 7.924 empresas de donde se seleccionó una muestra total final de 211 empresas. En la tabla 1 se muestran las características de las empresas que conforman la población para este estudio.

Se entregaron las encuestas con 35 preguntas a una población de 7,924 empresas. Los criterios para seleccionar esta población se basaron en el ranking Top 10,000 empresas (Canagh, 2015). Esta clasificación es la principal herramienta de información que existe sobre el mercado empresarial peruano e incluye textos de análisis, información financiera y datos de contacto de las principales empresas según el nivel de ingresos en el Perú. Las empresas incluidas dentro del ranking Top 10,000 empresas deben contar con una facturación superior al millón de soles, tener más de cinco años de fundación, tipo de operaciones y número de trabajadores.

De la población de 7,924 empresas se recibió respuesta efectiva de 211 empresas (la muestra) en territorio peruano. Los criterios de selección incluyeron ubicación geográfica, periodo de establecimiento, tipo de empresa según sus operaciones productivas, número de personas empleadas, entre otras. Las empresas son de bienes y servicios y todas son sociedades con fines de lucro. Cabe indicar que no hubo una definición de la población basada en su representatividad sino en la disponibilidad de los datos. 
Tabla 1. Características de las empresas incluidas en la población del estudio

\begin{tabular}{|c|c|}
\hline \multicolumn{2}{|c|}{ Descripción de la población } \\
\hline \multicolumn{2}{|c|}{ Tamaño de la empresa } \\
\hline Empresa grande (201 a más trabajadores) & $35,07 \%$ \\
\hline Empresa mediana $(51-200)$ & $40,76 \%$ \\
\hline Empresa pequeña $(11-50)$ & $21,33 \%$ \\
\hline Microempresa $(1-10)$ & $2,84 \%$ \\
\hline Total & $7,924(100 \%)$ \\
\hline Bienes & $37,44 \%$ \\
\hline Servicios & $62,56 \%$ \\
\hline
\end{tabular}

Fuente: elaboración propia.

La obtención del tamaño de la muestra se realizó con base en la población mencionada con un intervalo de confianza de $95 \%$ y un margen de error aproximado de 7.5 $\%$, lo que nos da un total de 211 empresas. La selección de la muestra se llevó a cabo usando un muestreo probabilístico aleatorio y para calcular el tamaño de la muestra se consideró la siguiente fórmula:

$$
\begin{gathered}
\mathrm{n}=\frac{N *\left(\propto_{\mathrm{c}} * 0.5\right)^{2}}{1+\left(e^{2} *(N-1)\right)} \\
\mathrm{n}=163
\end{gathered}
$$

Donde:

n: Tamaño de la muestra;

$\mathrm{N}$ : Tamaño de la población $(7,924$ empresas);

$\propto_{\mathrm{c} \text { : Valor correspondiente a la dis- }}$ tribución de Gauss (1,96 para un intervalo de confianza de $95 \%$ ); y

e: Margen de error permitido de 6.65

El cuestionario se realizó en los cuatros primeros meses de 2017 y se obtuvieron 211 respuestas voluntarias que se pudieron utilizar en el estudio. Del total de la muestra, el 39,4\% (83 empresas) posee certificación ISO 9001, mientras que el $60,6 \%$ restante (128 empresas) no están certificadas. El $65,41 \%$ de la muestra esta contestada por un Miembro del Directorio o por el Gerente General. El perfil del informante se describe en la tabla 2.

Del total de empresas encuestadas, el $24,17 \%$ son micro y pequeñas empresas, el 40,76\% son medianas empresas (de 51 a 200 trabajadores) y el 35,07\% son empresas grandes, es decir, centros de trabajo con más de 201 trabajadores. 
Las empresas de bienes conforman los siguientes sectores industriales: manufactura, conversión y reparaciones; las empresas de servicios conforman logística, seguridad y bienestar. En las empresas de manufactura, el alcance es en construcción, fabricación y ensamblaje y representan el 30,81\% de la muestra. En las empresas de conversión, su alcance incluye la extracción, transformación y reducción, representando el 8,53\% de la empresa. En las empresas de reparaciones se incluye el alcance de los servicios de reconstrucción, renovación y restauración, los cuales representan el 1,42\% de la muestra. En las empresas de logística, el alcance es el almacenamiento, transporte y comercio y representan el 33,18\% de la muestra. Por otro lado, en las empresas de seguridad, el alcance incluye los servicios de protección, financiamiento, defensa y orden, los cuales representan el 7,11 \% de la muestra. Finalmente, el alcance de las empresas de bienestar incluye salud, educación y asesoría, y representan el $18,96 \%$ de la muestra.

\section{INSTRUMENTO}

El cuestionario final incluye 35 preguntas acerca de la implementación de la TQM en las empresas nacionales e internacionales que están constituidas en Perú (Apéndice A). Se analizaron las preguntas del cuestionario calculando el Alfa de Cronbach para medir la confiabilidad asociada a la relación entre las preguntas de los factores evaluados (Apéndice B). Asimismo, se realizó la prueba no paramétrica U de MannWhitney, debido a las características de la distribución de la muestra (Apéndice C).

Tabla 2. Descripción de las empresas participantes en la muestra

\begin{tabular}{|l|c|}
\hline \multicolumn{1}{|c|}{ Descripción de las empresas } & $\begin{array}{c}\text { Año 2017 } \\
\text { Total }\end{array}$ \\
\hline Total de empresas (número de empresas) & $211(100 \%)$ \\
\hline Bienes & $86(40,75 \%)$ \\
\hline Empresas de Manufactura: construcción, fabricación y ensamblaje & $65(30,81 \%)$ \\
\hline Empresas de Conversión: extracción, transformación y reducción & $18(8,53 \%)$ \\
\hline Empresas de Reparaciones: reconstrucción, renovación y restauración & $3(1,42 \%)$ \\
\hline Servicios & $125(59,25 \%)$ \\
\hline
\end{tabular}




\begin{tabular}{|c|c|}
\hline Descripción de las empresas & $\begin{array}{c}\text { Año } 2017 \\
\text { Total }\end{array}$ \\
\hline Empresas de Logística: almacenamiento, transporte, comercio & $70(33,18 \%)$ \\
\hline Empresas de Seguridad: protección, financiamiento, defensa y orden & $15(7,11 \%)$ \\
\hline Empresas de Bienestar: salud, educación y asesoría & $40(18,96 \%)$ \\
\hline \multicolumn{2}{|l|}{ Tamaño de empresa (por número de trabajadores) } \\
\hline Empresa grande (201 a más) & $74(35,07 \%)$ \\
\hline Empresa mediana (51-200) & $86(40,76 \%)$ \\
\hline Empresa pequeña (11-50) & $45(21,33 \%)$ \\
\hline Microempresa (1-10) & $6(2,84 \%)$ \\
\hline \multicolumn{2}{|l|}{ Tiempo de fundación } \\
\hline Más de 20 años & $109(51,66 \%)$ \\
\hline 16 a 20 años & $48(22,75 \%)$ \\
\hline 11 a 15 años & $26(12,32 \%)$ \\
\hline 6 a 10 años & $28(13,27 \%)$ \\
\hline \multicolumn{2}{|l|}{ Persona que contestó } \\
\hline Miembro de Directorio & $12(5,69 \%)$ \\
\hline Presidente o Gerente General & $126(59,72 \%)$ \\
\hline Gerente de Área o Jefe de Departamento & $48(22,75 \%)$ \\
\hline Otro & $25(11,85 \%)$ \\
\hline
\end{tabular}

Fuente: elaboración propia.

La escala TQM utiliza los parámetros de la escala Likert, que consta de un conjunto de preguntas y está dividida en nueve factores: Liderazgo, Planeamiento de la calidad, Auditoría y evaluación de la calidad, Diseño del producto, Gestión de la calidad del proveedor, Con- trol y mejoramiento del proceso, Educación y entrenamiento, Círculos de la calidad y Enfoque hacia la satisfacción del cliente. La escala Likert es una escala comúnmente utilizada en cuestionarios, la cual fue desarrollada por Rensis Likert (Bertram, 2007). 


\section{ANÁLISIS DE LOS RESULTADOS}

En el Apéndice D se encuentran los resultados obtenidos, evidenciando si existen o no diferencias significativas en la TQM de las empresas en Perú, en función a la implementación de la ISO. Se obtuvieron diferencias significativas estadísticamente en los nueve factores entre empresas certificadas y empresas no certificadas con ISO 9001; además, se halló que el promedio en todos los factores es 3,55 en las empresas sin ISO 9001 y 3,91 para las empresas con ISO 9001.

La figura 4 muestra el comparativo de los resultados obtenidos por factores de las empresas certificadas y no certificadas con ISO 9001. Las empresas certificadas obtienen mayor valor que las que no lo están en todos los factores.

Figura 4. Comparación de factores en empresas con y sin certificación ISO 9001 en el 2016

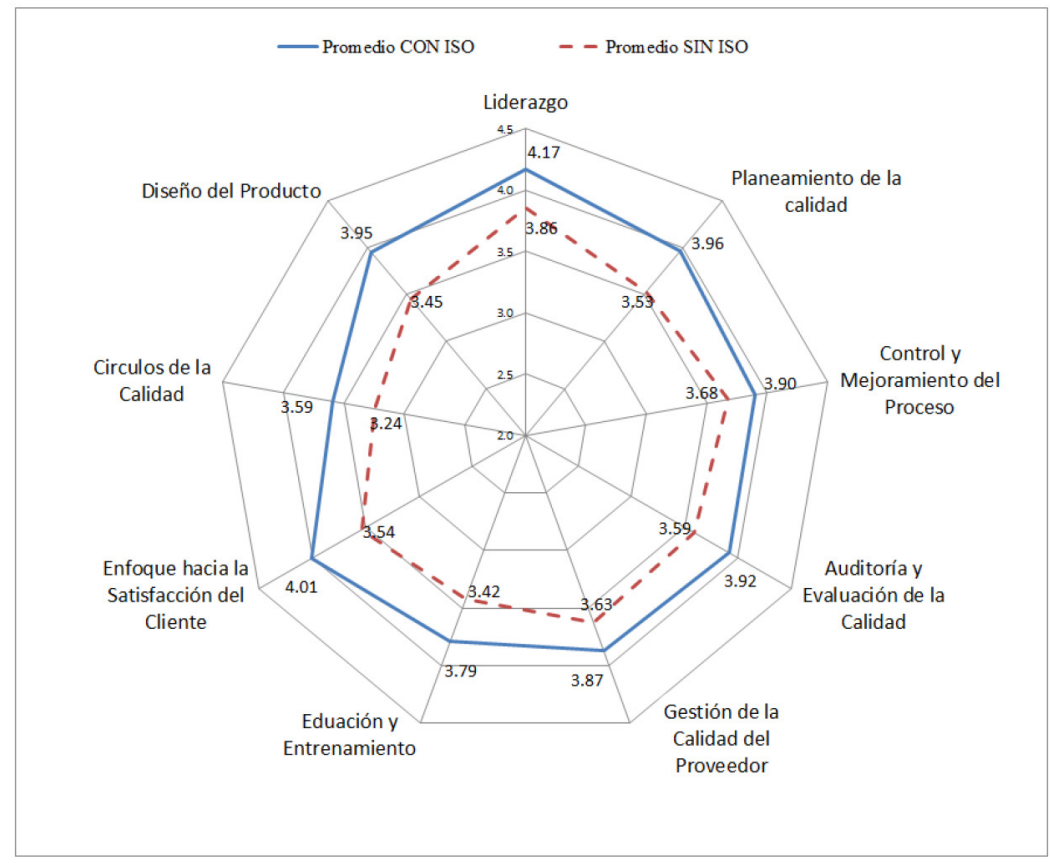

Fuente: elaboración propia 
En cuanto al Bloque de Alta Gerencia tenemos cuatro factores; en la muestra observamos que el promedio general obtenido por el factor de Liderazgo - Alta Gerencia $\left(X_{1}\right)$ es el más alto de los nueve factores estudiados $(3,98)$. La diferencia en los valores obtenidos entre empresas con ISO y empresas sin ISO es significativa en todas las variables del factor. Puede ser que esta diferencia significativa se deba a que los miembros de la Alta Gerencia en las empresas se involucran constantemente durante la implementación de todos los procesos relacionados a la gestión de la calidad. Esto incluye motivar de manera activa a los trabajadores para alentar su participación en estas actividades y asignar los recursos necesarios para lograr la implementación exitosa de estos procesos. Este factor está vinculado al principio "Liderazgo" de la norma ISO 9000:2015, en donde se indica que los líderes definen las condiciones en las que el personal se involucra para lograr los objetivos organizacionales en materia de calidad. Por consiguiente, siendo este factor el que obtiene los valores más altos, la alta gerencia ayuda al aumento de la eficiencia, a la mejora en la coordinación de los procesos organizacionales y en la comunicación. Algo notorio es que tanto las empresas certificadas como aquellas que no poseen certificación, buscan el éxito a largo plazo. Esto nos hace pensar que deben considerar los aspectos estratégicos para lograr los objetivos de sus empresas.

En la muestra, respecto al factor Planeamiento de calidad $\left(\mathrm{X}_{2}\right)$, las empresas con certificación ISO 9001 poseen un promedio superior y una diferencia significativa a las empresas sin la certificación en todas las variables del factor, siendo el promedio de 3,96 y 3,53 , respectivamente. Este resultado se debe a que la norma ISO 9001 requiere que se planifiquen y desarrollen políticas, objetivos y planes de calidad en una empresa. También exige que todo el personal participe en el proceso de desarrollo con la finalidad de ayudar a su percepción de la calidad a nivel organizacional e involucrar más los trabajadores en la elaboración de políticas y planes de calidad.

A través de la muestra observamos, en el factor Auditoría y evaluación de la calidad $\left(\mathrm{X}_{3}\right)$, se obtuvo una calificación de 3,92 en las empresas con certificación ISO, en comparación a un 3,59 de las empresas sin esta certificación. En este caso, la diferencia también es significativa y la razón puede ser que tanto las actividades de auditoría como las de evaluación de la calidad son requisitos primordiales para que una empresa 
pueda obtener el certificado de la norma ISO 9001. Asimismo, cabe resaltar que este factor está relacionado con el principio de toma de decisiones, según lo indican la evidencia y los datos objetivos, puesto que los procesos de toma de decisiones, la evaluación del desempeño del proceso, la eficiencia operativa y las capacidades de cuestionar y demostrar la eficacia de las decisiones mejoran de manera notable; sin embargo, se tiene que reforzar la práctica de realizar análisis comparativos (benchmarking).

\section{En el factor Diseño del producto} $\left(\mathbf{X}_{4}\right)$, el grupo de empresas con certificación ISO 9001 posee una diferencia significativa y un promedio superior de 3,95 en comparación con las empresas no certificadas que tienen un promedio de 3,45 . De acuerdo con esta comparación, las empresas que no están certificadas tienen que trabajar más en un método para desarrollar el producto. Este factor está relacionado al principio de enfoque al cliente, puesto que el objetivo es cumplir con sus requisitos y tratar de exceder sus expectativas con la finalidad de incrementar el valor y su satisfacción a través de un producto bien diseñado.

En el bloque del proveedor tenemos, según la muestra, para el factor Gestión y calidad del proveedor
$\left(\mathrm{X}_{5}\right)$, una diferencia significativa entre los valores obtenidos en este factor por empresas con ISO 9001 con respecto a las que no la tienen, siendo 3,87 y 3,63 , respectivamente. Este factor está relacionado con el principio de gestión de las relaciones el cual indica que para lograr un éxito constante, las empresas deben gestionar sus relaciones con las partes interesadas pertinentes, en este caso, los proveedores, ya que influyen en el desempeño de una empresa. De esta manera, aquellas organizaciones certificadas pueden incrementar su capacidad de crear valor para los proveedores y así asegurar una cadena de suministro bien gestionada. Gracias a la globalización y a los cambios que esta trae consigo, Perú ha logrado firmar varios convenios y tratados de libre comercio; por consiguiente, la relación con potenciales clientes y con los proveedores se convierte en un tema de suma importancia. De acuerdo con los resultados, se puede decir que las empresas con o sin certificación gestionan mejor sus relaciones con los proveedores y suministran productos con un nivel de calidad adecuado.

En el Bloque de Gestión de Procesos, para el factor Control y mejoramiento del proceso $\left(\mathrm{X}_{6}\right)$ se observa que las empresas con certificación ISO 9001 poseen una diferencia 
significativa en comparación con las empresas no certificadas. Este puntaje $(3,90)$ es el segundo más elevado de los nueve factores y esto puede explicar por qué las empresas certificadas lo relacionan al principio de "enfoque a procesos" de la norma ISO 9000:2015, que se basa en la obtención de resultados coherentes de manera más eficiente "cuando las actividades se entienden y gestionan como procesos interrelacionados que funcionan como un sistema coherente" (ISO, 2015, [en línea]). Esto ayuda a incrementar la capacidad de centrarse en los procesos más importantes, optimizar el desempeño, emplear de manera eficiente los recursos y aumentar la productividad mediante la mejora de la calidad de los procesos, que es un aspecto imprescindible para ser más competitivo. Asimismo, cabe recalcar que un sistema de gestión de calidad tiene que tener procesos que estén interrelacionados y la organización tiene la obligación de entender cómo este sistema produce los resultados necesarios para optimizar el sistema y desempeño organizacional. En contraste, aquellas empresas sin certificación tienen un menor nivel de percepción en esta materia. Aquellas empresas sin la certificación, cuyo promedio es 3,68, también tienen este factor como el segundo en importancia; en general, se tiene que mejorar en el uso de las herramientas que ayuden a mejorar la calidad en los procesos.

En la muestra se observa que, en el factor Educación y entrenamiento $\left(\mathrm{X}_{7}\right)$, la variable sobre si los empleados de la empresa se encuentran activamente involucrados en las actividades relacionadas con la calidad posee un promedio de 3,79 en empresas con certificación y 3,42 en empresas sin certificación. En este factor también existe una diferencia significativa, que podría deberse a que en las empresas con la certificación ISO 9001 se le da una mayor relevancia a la capacitación en materia de calidad a los trabajadores para que puedan emplear las herramientas adecuadas y así logren realizar una mejor gestión de la calidad dentro de la organización. Gracias a esto, el personal participa de manera activa en las actividades de la calidad y por eso tienen una mejor conciencia con respecto a la cultura de calidad de la empresa. De igual manera, se tiene que capacitar al personal en el uso de herramientas para la gestión de la calidad.

En la muestra, el factor Círculos de calidad $\left(\mathrm{X}_{8}\right)$ posee un promedio de 3,59 en empresas con certificación ISO 9001 y 3,24 sin certificación, $y$ tienen una diferencia significativa que podría estar influenciada por el principio de mejora que tiene un 
enfoque continuo hacia "la mejora del desempeño del proceso, de las capacidades de la organización y de la satisfacción del cliente" (ISO, 2015, [en línea]) y por la promoción de la innovación dentro de la empresa, la cual se incrementa gracias a la implementación de este factor dentro de la organización. Tanto las empresas certificadas como las no certificadas tienen este factor como el más bajo de los nueve. Fabl y Álvarez (211) afirmaron que "el éxito o fracaso de los círculos de calidad puede ser causado por un conjunto de condiciones ligadas a su entorno organizacional". Algunas de estas condiciones pueden ser el tipo de industria, la competencia, el estilo de gestión, etc. Esto podría explicar por qué los círculos de calidad, como práctica de gestión, no están muy arraigados dentro de las organizaciones. Por esta razón, las empresas deben incentivar la realización de los círculos de calidad para que ayude a mejorar el trabajo en equipo.

Finalmente, en el Bloque del Cliente, el factor Enfoque hacia la satisfacción del cliente $\left(\mathrm{X}_{9}\right)$, según la muestra observada, obtiene un promedio de 4,01 para las empresas con certificación ISO y un valor de 3,54 en las empresas sin la certificación. En este factor también se tiene una diferencia significativa. Esto se debe a que dicho factor está relacionado con el principio de enfoque al cliente de la ISO 9001, ya que el éxito de una empresa yace en atraer y conservar la confianza de los clientes para tener la oportunidad de crear valor para ellos. Asimismo, se debe prestar atención a sus quejas, porque esto ayuda a incrementar su satisfacción $y$, por ende, a fidelizarlos.

\section{CONCLUSIONES}

El presente estudio es una continuación de la encuesta realizada a las empresas peruanas en 2011 (Benzaquen, 2013). En esta ocasión, cabe recalcar que en ambas muestras de 2011 y 2017 la mayoría son empresas de servicios (55\% y $59 \%$, respectivamente). La muestra de 211 empresas en el presente estudio está conformada, en su mayoría, por empresas de logística $(33,18 \%)$, y por medianas empresas con 51 a 200 trabajadores (40,76\%).

Al analizar el nivel de calidad en la presente muestra de las empresas peruanas, en función a los nueve factores de TQM, se encontraron diferencias significativas en todos los factores entre las empresas certificadas con ISO 9001 y las que no poseen dicha certificación. Es importante mencionar que del total de la muestra, el número de empresas sin certificación ISO es mayor 
(128) al número de empresas certificadas (83). Esto se puede deber a que en la actualidad muchas empresas se encuentran en proceso de migración al ISO 9001:2015.

Los resultados confirman que las empresas con certificación ISO 9001 tienen mejor promedio en los nueve factores de TQM analizados en la presente investigación, lo que enfatiza y confirma la importancia de la certificación para mejorar la gestión de la calidad en las empresas peruanas. De los nueve factores, los de mayor promedio en las empresas con certificación fueron: Liderazgo-Alta Gerencia, Planeamiento de la calidad y Diseño del producto, con valores de 4,17, 3,96 y 3,95 , respectivamente. Estos factores están estrechamente relacionados con los principios de Liderazgo y enfoque al cliente de la norma ISO 9000:2015, porque el objetivo primordial es establecer las condiciones para lograr los objetivos organizacionales y cumplir con los requisitos del cliente a fin de exceder sus expectativas (ISO, 2015). Los líderes de las organizaciones son considerados como los responsables de implementar y asegurar que la gestión de la calidad en la empresa se lleve a cabo de manera sostenida a través del tiempo, por esta razón, las empresas certificadas tienen un mayor interés en estos factores, porque son exigencias que deben cumplir para obtener la certificación.

Tanto las empresas certificadas como las que no lo están tienen un promedio bastante bajo en el factor de Círculos de calidad, al igual que en la encuesta realizada en 2011, esto quiere decir que aún no hay una mejora en la provisión de capacitaciones adecuadas para usar de manera correcta este factor y así mejorar la gestión de la calidad.

Todas las empresas tienden a buscar resultados a largo plazo, por eso consideran los aspectos estratégicos y evalúan constantemente los productos suministrados por el proveedor para que los procesos operativos cumplan con los plazos de entrega solicitados por los clientes.

Los resultados de esta investigación pueden alentar a las empresas peruanas a asignar los recursos necesarios para que se embarquen en el proceso de obtención de la certificación ISO 9001 en la versión del 2015, debido al impacto favorable que tiene esta certificación en la calidad de sus procesos y productos y, por lo tanto, en la satisfacción de sus clientes. Esto es muy importante, puesto que según el INACAL (2016), solo un $1 \%$ de las empresas formales del Perú cuenta con sistemas de gestión de calidad implementados. 
Por último, la metodología utilizada en el presente estudio se puede usar en otros países sudamericanos y así realizar comparaciones entre los países con la finalidad de identificar los puntos fuertes y débiles en materia de calidad. De esta manera, se pueden desarrollar oportunidades de mejora dentro de los sistemas de gestión de la calidad de las empresas y ejecutar planes de acción para superar las brechas o debilidades.

\section{REFERENCIAS}

(julio, 2014). Gobierno plantea crear Sistema Nacional de Calidad que aportará US\$ 234 millones anuales. Gestión. Recuperado de http:/gestion.pe/ economia/gobierno-planteacrear-sistema-nacional-calidadque-aportara-us-234-millonesanuales-2101804

Aba, E. K., Badar, M. A., \& Hayden, M. A. (2016). Impact of ISO 9001 Certification on Firms Financial Operating Performance. The International Journal of Quality \& Reliability Management, 33(1), 78-89. Recuperado de https://search.proquest.com/ docview/1752574262?account id $=28391$

Androniceanu, A. (2017). The Three-Dimensional Approach of Total Quality Management, an Essential Strategic Option for Business Excellence. Amfiteatru Economic, 19(44), 61-78.

Al-Khalili, A.; \& Subari, K. (2014). The Interrelationship between ISO 9000 and Total Quality Management: An Empirical Investigation with a Focus on Soft and Hard TQM Dimensions. Int. J. Services and Operations Management, 18(4), 429-448.

American Society for Quality [ASQ]. (s.f.). Quality glossary. Recuperado de http://asq.org/glossary/q. html

Antony, J., Leung K., \& Knowless, G. (2002). Critical Success Factors of TQM Implementation in Hong Kong Industries. International Journal of Quality $y$ Reliability Management, 19(5), 551-566.

Arumugam, V., Ooi, K.B., \& Fong, T.C (2008). TQM Practices and Quality Management Performance: An Investigation of their Relationship Using Data from ISO 9001:2000 Firms in Malaysia. The TQM Journal, 20(6), 636-650.

Báez, L. (2013). Mejoramiento de la gestión pública con ISO 9001:2008.Estudiodecaso.Scientia et Technica, 18(1), 126-131. Benzaquen, J., \& Convers, J. (2016). El ISO 9001 y TQM en las empresas de Ecuador. Globalización, Competitividad y Gobernabilidad, 10(3), 153-176. 
Benzaquen, J., \& Convers, J. (2015). El ISO 9001 y TQM en las empresas de Colombia. Globalización, Competitividad y Gobernabilidad, 9(3), 107-128.

Benzaquen, J. (2014). La Ansiada Calidad Total. Revista Strategia, 34, 54-55.

Benzaquen, J. (2013). Calidad en las empresas latinoamericanas: El caso peruano. Globalización, Competitividad y Gobernabilidad, 7(1), 41-59.

Bertram, D. (2013). Likert Scale is the Meaning of Life. Yugoslavia: University of Belgrade. Recuperado de http://poincare.matf. bg.ac.rs/ kristina/topic-danelikert.pdf

Bohórquez, L. E. (2010). Estudio del impacto de la certificación ISO 9001: 2000 en la competitividadpaís. Ingeniería, 15(1), 22-34.

Canagh, J. (2015). Perú: The TOP 10000 Companies 2015. Lima: Punto \& Grafía.

Centro de Desarrollo Industrial [CDI]. (2011). Semana de la calidad. Recuperado de http://www. cdi.org.pe/semana_cgc.htm

Centro de Desarrollo Industrial [CDI]. (s.f.) Servicios. Recuperado de http://www.cdi.org.pe/ default.html

Fabl, B., \& Álvarez, C. (2011). Los Círculos de Calidad. Lecciones de una experiencia internacional. Cuadernos de Administración, 13(19), 25-38.
George, D., \& Mallery, P. (2003). SPSS for Windows Step by Step: A Simple Guide and Reference 11.0 update (4 Ed.). Boston, MA: Allyn and Bacon.

Huq, Z., \& Stolen, J. D. (1998). Total Quality Management Contrasts in Manufacturing and Service Industries. International Journal of Quality and Reliability Management, 15(2), 138-161. Indecopi (2010). Certificación ISO 9001. Recuperado de https:// www.indecopi.gob.pe/repositorioaps/0/14/jer/guias_informativas/iso9001.pdf

Instituto Nacional de Calidad. (2014). Sistema Nacional de Calidad. Recuperado de http://www. inacal.gob.pe/principal/categoria/ sistema-nacional-de-calidad

Instituto Nacional de Calidad. (2016). Solo $1 \%$ de empresas en el Perú cuenta con Sistemas de Gestión de Calidad. Recuperado de http://www.inacal.gob.pe/ principal/noticia/solo-de-empresas-en-el-peru

Miranda, F., \& Chamorro, A. (2007). Introducción a la Gestión de la Calidad. Madrid: Delta Publicaciones.

Miranda, R. (2002). Enfoque del Programa Gestión de la Calidad Institucional. Heredia: Universidad Nacional de Costa Rica.

Miroslav, K., Slobodan, Č., Vladimir, D., \& Tijana, R. (2017). Total 
Quality Management Implementation and Guest Satisfaction in Hospitality. Amfiteatru Economic, 19(44), 124-143.

Mundaca, M., Rojas, E., Pezo, G., Chú, R., \& Carrillo, P. (2003). Causas de la Crisis Empresarial Peruana: Un Modelo de Gestión en Entornos Complejos. Recuperado de https:/ezapatarunp. files.wordpress.com/2011/09/ see-problematica-empresarial-peruana.pdf

Nunnally, J. (1978). Psychometric Theory. New York, NY: McGraw-Hill.

Organización Internacional de Normalización. (2008). Norma Internacional ISO 9001. Sistemas de gestión de la calidad. Génova: ISO.

Organización Internacional de Normalización. (2015). Norma Internacional ISO 9001:2015. Sistemas de gestión de la calidad. Génova: ISO.

Organización Internacional de Normalización. (2017). ISO Survey of certifications to management system standards. Recuperado de: http://isotc.iso.org/livelink/li velink? func $=11 \&$ objId $=1880877$ $2 \&$ objAction $=$ browse \&viewTy $\mathrm{pe}=1$

Sánchez, E. (2005). Seis sigma, filosofia de gestión de la calidad: estudios teóricos y su posible aplicación en el Perú. (Tesis de grado). Universidad de Piura, Piura. Recuperado de http://pirhua.udep.edu.pe/bitstream/handle/123456789/1213/ING_427. pdf?sequence $=1$

Sociedad Nacional de Industria. (2014). Decreto supremo que aprueba la política nacional para la calidad (Decreto Supremo $N .^{\circ}$ 046-2014-PCM). Recuperado de http://www2.sni.org. pe/servicios/legal/reportelegal/ content/view/12262/27/

Sociedad Nacional de Industrias [SNI]. (s.f.). Nuestra historia. Recuperado de http://www.sni. org.pe/?page_id $=98$

Solo el 1\% de empresas en Perú cuenta con sistemas de gestión de calidad. (2016, Julio 6). RPP Noticias. Recuperado de http://rpp. pe/campanas/branded-content/ solo-el-1-de-empresas-en-perucuenta-con-sistemas-de-gestionde-calidad-noticia-977089

Velázquez, J., Terraza, R., \& Ruiz, L. (2014). El sistema de gestión de calidad bajo las normas ISO 9001:2008 como elemento articulador de los lineamientos de la evaluación y acreditación de programas educativos de ciencias sociales y administrativa de la Universidad Estatal de Sonora. Global Conference on Business \& Finance Proceedings, 9(1), 1213-1226. 


\section{ANEXOS}

\section{Anexo 1. Preguntas de los Factores de la Calidad}

\begin{tabular}{|c|c|}
\hline Factores de la Calidad & Variables (preguntas) \\
\hline \multirow{5}{*}{ Alta Gerencia $-\mathrm{X}_{1}$} & $\begin{array}{l}\text { La alta gerencia participa activamente en la Gestión de la Calidad en la } \\
\text { empresa }-\mathbf{X}_{11}\end{array}$ \\
\hline & $\begin{array}{l}\text { La alta gerencia de la empresa alienta firmemente la participación de los } \\
\text { empleados en la Gestión de la Calidad }-\mathbf{X}_{12}\end{array}$ \\
\hline & $\begin{array}{l}\text { La alta gerencia de la empresa se reúne de manera regular para discutir } \\
\text { temas relacionados con la Gestión de la Calidad }-\mathbf{X}_{13}\end{array}$ \\
\hline & $\begin{array}{l}\text { La alta gerencia de la empresa proporciona los recursos apropiados para } \\
\text { elevar el nivel de la calidad }-\mathbf{X}_{14}\end{array}$ \\
\hline & La alta gerencia busca el éxito de la empresa a largo plazo $-\mathbf{X}_{15}$ \\
\hline \multirow{3}{*}{$\begin{array}{l}\text { Planeamiento de la } \\
\text { calidad }-\mathrm{X}_{2}\end{array}$} & $\begin{array}{l}\text { La empresa tiene metas específicas y detalladas en cuanto a la calidad } \\
-\mathbf{X}_{21}\end{array}$ \\
\hline & $\begin{array}{l}\text { La empresa presta atención al cumplimiento y éxito de sus políticas y } \\
\text { planes relacionados con la calidad }-\mathbf{X}_{22}\end{array}$ \\
\hline & $\begin{array}{l}\text { La empresa involucra a sus empleados para hacer las políticas y planes } \\
\text { de calidad }-\mathbf{X}_{23}\end{array}$ \\
\hline \multirow{3}{*}{$\begin{array}{l}\text { Auditoría y evaluación } \\
\text { de la calidad }-\mathrm{X}_{3}\end{array}$} & La empresa evalúa regularmente sus políticas y planes de la calidad $-\mathbf{X}_{31}$ \\
\hline & El benchmarking se utiliza ampliamente en la empresa $-\mathbf{X}_{32}$ \\
\hline & La empresa obtiene datos objetivos para la toma de decisiones $-\mathbf{X}_{33}$ \\
\hline \multirow{3}{*}{$\begin{array}{l}\text { Diseño del producto } \\
\qquad-\mathrm{X}_{4}\end{array}$} & $\begin{array}{l}\text { Los requerimientos de los clientes son plenamente considerados en el } \\
\text { diseño del producto }-\mathbf{X}_{41}\end{array}$ \\
\hline & La empresa invierte en el diseño del producto $-\mathbf{X}_{42}$ \\
\hline & La empresa tiene un método para desarrollar el diseño del producto $-\mathbf{X}_{43}$ \\
\hline \multirow{3}{*}{$\begin{array}{l}\text { Gestión de la calidad del } \\
\text { proveedor }-\mathrm{X}_{5}\end{array}$} & $\begin{array}{l}\text { La empresa ha establecido relaciones de cooperación a largo plazo con } \\
\text { sus proveedores }-\mathbf{X}_{51}\end{array}$ \\
\hline & $\begin{array}{l}\text { La empresa posee información detallada acerca del desempeño de los } \\
\text { proveedores en cuanto a calidad }-\mathbf{X}_{52}\end{array}$ \\
\hline & $\begin{array}{l}\text { La calidad de los productos que los proveedores suministran a la empresa } \\
\text { es adecuada }-\mathbf{X}_{53} \\
\text { La empresa realiza auditorías o evaluaciones de sus proveedores }-\mathbf{X}_{54}\end{array}$ \\
\hline
\end{tabular}




\begin{tabular}{|c|c|}
\hline Factores de la Calidad & Variables (preguntas) \\
\hline $\begin{array}{l}\text { Control y mejoramiento } \\
\text { de proceso }-\mathrm{X}_{6}\end{array}$ & $\begin{array}{l}\text { El proceso operativo en la empresa satisface los requerimientos de plazo } \\
\text { de entrega de los clientes }-\mathbf{X}_{61} \\
\text { Las instalaciones y la disposición física del equipo operativo en la } \\
\text { empresa funcionan apropiadamente }-\mathbf{X}_{62} \\
\text { Los equipos operativos de la empresa reciben buen mantenimiento }-\mathbf{X}_{63} \\
\text { La empresa utiliza las siete herramientas de Control de la Calidad para } \\
\text { el control y mejoramiento del proceso (Diagrama de Flujo, Diagrama de } \\
\text { Ishikawa o Causa - Efecto, Lista de Verificación, Diagrama de Pareto, } \\
\text { Histograma, Gráficos de Control, Diagrama de Relaciones) }-\mathbf{X}_{64} \\
\text { La empresa implementa el control de calidad con eficacia }-\mathbf{X}_{65}\end{array}$ \\
\hline \multirow{4}{*}{$\begin{array}{l}\text { Educación } \\
\text { entrenamiento }-\mathrm{X}_{7}\end{array}$} & $\begin{array}{l}\text { La mayoría de empleados de la empresa reciben educación y } \\
\text { entrenamiento en cuanto a calidad }-\mathbf{X}_{71}\end{array}$ \\
\hline & $\begin{array}{l}\text { La mayoría de los empleados de la empresa son capaces de utilizar las } \\
\text { herramientas para la gestión de la calidad }-\mathbf{X}_{72}\end{array}$ \\
\hline & $\begin{array}{l}\text { Los empleados de la empresa se encuentran activamente involucrados en } \\
\text { las actividades relacionadas con la calidad }-\mathbf{X}_{73}\end{array}$ \\
\hline & $\begin{array}{l}\text { La conciencia de los trabajadores de la empresa hacia la calidad es fuerte } \\
-\mathbf{X}_{74}\end{array}$ \\
\hline \multirow{4}{*}{ Círculos de calidad $-\mathrm{X}_{8}$} & La empresa está capacitada para realizar círculos de calidad - $\mathbf{X}_{\mathbf{8 1}}$ \\
\hline & $\begin{array}{l}\text { La mayoría de los empleados de la empresa realiza actividades de } \\
\text { círculos de calidad }-\mathbf{X}_{\mathbf{8 2}}\end{array}$ \\
\hline & $\begin{array}{l}\text { Se utilizan las herramientas adecuadas para realizar los círculos de } \\
\text { calidad en la empresa }-\mathbf{X}_{\mathbf{8 3}}\end{array}$ \\
\hline & La empresa ha obtenido ahorros por los círculos de calidad $-\mathbf{X}_{\mathbf{8 4}}$ \\
\hline \multirow{4}{*}{$\begin{array}{l}\text { Enfoque hacia la } \\
\text { satisfacción del cliente } \\
-X_{9}\end{array}$} & $\begin{array}{l}\text { La empresa cuenta con medios para obtener información sobre los } \\
\text { clientes }-\mathbf{X}_{91}\end{array}$ \\
\hline & $\begin{array}{l}\text { La empresa lleva a cabo una encuesta de satisfacción del cliente todos } \\
\text { los años }-\mathbf{X}_{\mathbf{9 2}}\end{array}$ \\
\hline & $\begin{array}{l}\text { El personal de todos los niveles de la empresa presta atención a la } \\
\text { información sobre las quejas de los clientes }-\mathbf{X}_{93}\end{array}$ \\
\hline & $\begin{array}{l}\text { La empresa realiza una evaluación general de los requerimientos de los } \\
\text { clientes }-\mathbf{X}_{\mathbf{9 4}}\end{array}$ \\
\hline
\end{tabular}


Anexo 2. Resultados del Coeficiente de Alpha de Cronbach

\begin{tabular}{|l|c|c|}
\hline \multicolumn{1}{|c|}{ Factor } & Alpha de Cronbach & N. $^{\circ}$ \\
\hline$X_{1}$ Alta Gerencia & 0.8922 & 5 \\
\hline$X_{2}$ Planeamiento de la Calidad & 0.8197 & 3 \\
\hline$X_{3}$ Auditoria y Evaluación de la Calidad & 0.7195 & 3 \\
\hline$X_{4}$ Diseño del Producto & 0.8745 & 3 \\
\hline$X_{5}$ Gestión de la Calidad del Proveedor & 0.6704 & 4 \\
\hline$X_{6}$ Control y Mejoramiento del Proceso & 0.7844 & 5 \\
\hline$X_{7}$ Educación y Entrenamiento & 0.8297 & 4 \\
\hline$X_{8}$ Círculos de la Calidad & 0.8455 & 4 \\
\hline$X_{9}$ Enfoque hacia la Satisfacción del Cliente & 0.7115 & 4 \\
\hline
\end{tabular}

Notas. El orden de las preguntas no guarda correlación con el orden consecutivo de los factores que se midieron, por lo que existe mayor confiabilidad y validez del instrumento que si las preguntas estuviesen agrupadas correlativamente para cada factor evaluado.
George y Mallery (2003) indicaron que un resultado del Alpha de Cronbach es más que aceptable si es mayor a 0.7 . No obstante, Nunnally (1978) determinó que la medida del Alpha de Cronbach puede ser aceptada cuando es mayor a 0.6. 


\section{Anexo 3. Resumen de la prueba U de Mann-Whitney para heteroge- neidad muestras independientes}

\begin{tabular}{|l|c|}
\hline \multicolumn{1}{|c|}{ Factor } & Sig. asintótica (bilateral) \\
\hline Alta Gerencia & 0.001 \\
\hline Planeamiento de la Calidad & 0.000 \\
\hline Auditoria y Evaluación de la Calidad & 0.000 \\
\hline Diseño del Producto & 0.000 \\
\hline Gestión de la Calidad del Proveedor & 0.007 \\
\hline Control y Mejoramiento del Proceso & 0.005 \\
\hline Educación y Entrenamiento & 0.000 \\
\hline Círculos de la Calidad & 0.001 \\
\hline Enfoque hacia la Satisfacción del Cliente & 0.000 \\
\hline
\end{tabular}

Nota. ${ }^{*} p<0.05=$ diferencia significativa en la calificación a un nivel de error de $0.05 \%$

$* p>0.05=$ no existen diferencias significativas 


\section{Anexo 4. Valores promedios por factor}

\begin{tabular}{|c|c|c|c|}
\hline & $\begin{array}{l}\text { Empresas } \\
\text { encuestadas } \\
\quad(211)\end{array}$ & $\begin{array}{l}\text { Empresas } \\
\text { con ISO } \\
(83)\end{array}$ & $\begin{array}{c}\text { Empresas } \\
\text { sin ISO } \\
(128)\end{array}$ \\
\hline Alta Gerencia - Liderazgo $-X_{1}$ & 3.98 & 4.17 & 3.86 \\
\hline $\begin{array}{l}\text { La alta gerencia participa activamente en la Gestión de la } \\
\text { Calidad en la empresa }-\mathbf{X}_{11}\end{array}$ & 3.95 & 4.13 & 3.83 \\
\hline $\begin{array}{l}\text { La alta gerencia de la empresa alienta firmemente la } \\
\text { participación de los empleados en la Gestión de la Calidad } \\
-\mathbf{X}_{12}\end{array}$ & 3.93 & 4.18 & 3.78 \\
\hline $\begin{array}{l}\text { La alta gerencia de la empresa se reúne de manera regular } \\
\text { para discutir temas relacionados con la Gestión de la Calidad } \\
-\mathbf{X}_{13}\end{array}$ & 3.67 & 3.85 & 3.56 \\
\hline $\begin{array}{l}\text { La alta gerencia de la empresa proporciona los recursos } \\
\text { apropiados para elevar el nivel de la calidad }-\mathbf{X}_{14}\end{array}$ & 3.87 & 4.18 & 3.67 \\
\hline $\begin{array}{l}\text { La alta gerencia busca el éxito de la empresa a largo plazo - } \\
\mathbf{X}_{15}\end{array}$ & 4.48 & 4.52 & 4.46 \\
\hline Planeamiento de Calidad $-\mathrm{X}_{2}$ & 3.70 & 3.96 & 3.53 \\
\hline $\begin{array}{l}\text { La empresa tiene metas específicas y detalladas en cuanto a } \\
\text { la calidad }-\mathbf{X}_{21}\end{array}$ & 3.88 & 4.20 & 3.67 \\
\hline $\begin{array}{l}\text { La empresa presta atención al cumplimiento y éxito de sus } \\
\text { políticas y planes relacionados con la calidad }-\mathbf{X}_{22}\end{array}$ & 3.67 & 3.98 & 3.47 \\
\hline $\begin{array}{l}\text { La empresa involucra a sus empleados para hacer las políticas } \\
\text { y planes de calidad }-\mathbf{X}_{23}\end{array}$ & 3.55 & 3.78 & 3.40 \\
\hline Auditoría y evaluación de la calidad $-\mathrm{X}_{3}$ & 3.72 & 3.92 & 3.59 \\
\hline $\begin{array}{l}\text { La empresa evalúa regularmente sus políticas y planes de la } \\
\text { calidad }-\mathbf{X}_{31}\end{array}$ & 3.70 & 4.00 & 3.50 \\
\hline El benchmarking se utiliza ampliamente en la empresa $-\mathbf{X}_{32}$ & 3.55 & 3.74 & 3.42 \\
\hline $\begin{array}{l}\text { La empresa obtiene datos objetivos para la toma de } \\
\text { decisiones }-\mathbf{X}_{33}\end{array}$ & 3.91 & 4.06 & 3.82 \\
\hline Diseño del producto $-\mathrm{X}_{4}$ & 3.65 & 3.95 & 3.45 \\
\hline $\begin{array}{l}\text { Los requerimientos de los clientes son plenamente } \\
\text { considerados en el diseño del producto }-\mathbf{X}_{41}\end{array}$ & 3.82 & 4.07 & 3.66 \\
\hline La empresa invierte en el diseño del producto $-\mathbf{X}_{42}$ & 3.61 & 3.91 & 3.41 \\
\hline
\end{tabular}




\begin{tabular}{|c|c|c|c|}
\hline & \\
\hline & $\begin{array}{c}\text { Empresas } \\
\text { encuestadas } \\
(211)\end{array}$ & $\begin{array}{c}\text { Empresas } \\
\text { con ISO } \\
(83)\end{array}$ & $\begin{array}{c}\text { Empresas } \\
\text { sin ISO } \\
(128)\end{array}$ \\
\hline $\begin{array}{l}\text { La empresa tiene un método para desarrollar el diseño del } \\
\text { producto }-\mathbf{X}_{43}\end{array}$ & 3.52 & 3.88 & 3.29 \\
\hline Gestión de la Calidad del Proveedor $-\mathrm{X}_{5}$ & 3.73 & 3.87 & 3.63 \\
\hline $\begin{array}{l}\text { La empresa ha establecido relaciones de cooperación a largo } \\
\text { plazo con sus proveedores }-\mathbf{X}_{51}\end{array}$ & 3.81 & 3.84 & 3.79 \\
\hline $\begin{array}{l}\text { La empresa posee información detallada acerca del } \\
\text { desempeño de los proveedores en cuanto a calidad }-\mathbf{X}_{52}\end{array}$ & 3.58 & 3.72 & 3.49 \\
\hline $\begin{array}{l}\text { La calidad de los productos que los proveedores suministran } \\
\text { a la empresa es adecuada }-\mathbf{X}_{53}\end{array}$ & 4.04 & 4.04 & 4.05 \\
\hline $\begin{array}{l}\text { La empresa realiza auditorías o evaluaciones de sus } \\
\text { proveedores }-\mathbf{X}_{54}\end{array}$ & 3.47 & 3.89 & 3.21 \\
\hline Control y Mejoramiento del Proceso $-\mathrm{X}_{6}$ & 3.77 & 3.90 & 3.68 \\
\hline $\begin{array}{l}\text { El proceso operativo en la empresa satisface los } \\
\text { requerimientos de plazo de entrega de los clientes }-\mathbf{X}_{61}\end{array}$ & 3.91 & 3.89 & 3.92 \\
\hline $\begin{array}{l}\text { Las instalaciones y la disposición física del equipo operativo } \\
\text { en la empresa funcionan apropiadamente }-\mathbf{X}_{62}\end{array}$ & 3.98 & 4.02 & 3.95 \\
\hline $\begin{array}{l}\text { Los equipos operativos de la empresa reciben buen } \\
\text { mantenimiento }-\mathbf{X}_{63}\end{array}$ & 3.94 & 3.93 & 3.95 \\
\hline $\begin{array}{l}\text { La empresa utiliza las siete herramientas de Control de } \\
\text { la Calidad para el control y mejoramiento del proceso } \\
\text { (Diagrama de Flujo, Diagrama de Ishikawa o Causa - Efecto, } \\
\text { Lista de Verificación, Diagrama de Pareto, Histograma, } \\
\text { Gráficos de Control, Diagrama de Relaciones) - } \mathbf{X}_{64}\end{array}$ & 3.18 & 3.51 & 2.96 \\
\hline $\begin{array}{l}\text { La empresa implementa el control de calidad con eficacia - } \\
\mathbf{X}_{65}\end{array}$ & 3.83 & 4.13 & 3.64 \\
\hline Educación y Entrenamiento $-\mathrm{X}_{7}$ & 3.57 & 3.79 & 3.42 \\
\hline $\begin{array}{l}\text { La mayoría de empleados de la empresa reciben educación y } \\
\text { entrenamiento en cuanto a calidad }-\mathbf{X}_{71}\end{array}$ & 3.47 & 3.83 & 3.25 \\
\hline $\begin{array}{l}\text { La mayoría de los empleados de la empresa son capaces de } \\
\text { utilizar las herramientas para la gestión de la calidad }-\mathbf{X}_{72}\end{array}$ & 3.48 & 3.68 & 3.35 \\
\hline $\begin{array}{l}\text { Los empleados de la empresa se encuentran activamente } \\
\text { involucrados en las actividades relacionadas con la calidad } \\
-\mathbf{X}_{73}\end{array}$ & 3.72 & 3.88 & 3.62 \\
\hline
\end{tabular}




\begin{tabular}{|l|c|c|c|}
\cline { 2 - 4 } \multicolumn{1}{l|}{} & $\begin{array}{c}\text { Empresas } \\
\text { encuestadas } \\
(211)\end{array}$ & $\begin{array}{c}\text { Empresas } \\
\text { con ISO } \\
(83)\end{array}$ & $\begin{array}{c}\text { Empresas } \\
\text { sin ISO } \\
(128)\end{array}$ \\
\hline $\begin{array}{l}\text { La conciencia de los trabajadores de la empresa hacia la } \\
\text { calidad es fuerte }-\mathbf{X}_{74}\end{array}$ & 3.60 & 3.80 & 3.47 \\
\hline Círculos de la Calidad $-\mathbf{X}_{8}$ & 3.38 & 3.59 & 3.24 \\
\hline $\begin{array}{l}\text { La empresa está capacitada para realizar círculos de calidad } \\
-\mathbf{X}_{\mathbf{8 1}}\end{array}$ & 3.59 & 3.84 & 3.43 \\
\hline $\begin{array}{l}\text { La mayoría de los empleados de la empresa realiza } \\
\text { actividades de círculos de calidad }-\mathbf{X}_{\mathbf{8 2}}\end{array}$ & 3.13 & 3.32 & 3.02 \\
\hline $\begin{array}{l}\text { Se utilizan las herramientas adecuadas para realizar los } \\
\text { círculos de calidad en la empresa }-\mathbf{X}_{83}\end{array}$ & 3.40 & 3.66 & 3.23 \\
\hline $\begin{array}{l}\text { La empresa ha obtenido ahorros por los círculos de calidad } \\
-\mathbf{X}_{84}\end{array}$ & 3.39 & 3.59 & 3.26 \\
\hline Enfoque hacia la Satisfacción del Cliente $-\mathbf{X}_{9}$ & 3.73 & 4.01 & 3.54 \\
\hline $\begin{array}{l}\text { La empresa cuenta con medios para obtener información } \\
\text { sobre los clientes }-\mathbf{X}_{\mathbf{9 1}}\end{array}$ & 3.79 & 3.95 & 3.69 \\
\hline $\begin{array}{l}\text { La empresa lleva a cabo una encuesta de satisfacción del } \\
\text { cliente todos los años }-\mathbf{X}_{\mathbf{9 2}}\end{array}$ & 3.59 & 4.22 & 3.19 \\
\hline $\begin{array}{l}\text { El personal de todos los niveles de la empresa presta atención } \\
\text { a la información sobre las quejas de los clientes }-\mathbf{X}_{93}\end{array}$ & 3.71 & 3.84 & 3.62 \\
\hline $\begin{array}{l}\text { La empresa realiza una evaluación general de los } \\
\text { requerimientos de los clientes }-\mathbf{X}_{94}\end{array}$ & 3.82 & 4.06 & 3.67 \\
\hline Valor Promedio (Y) & 3.69 & 3.91 & 3.55 \\
\hline
\end{tabular}

Nota. Los Valores del 1 al 5 significan lo siguiente:

1.-Totalmente en desacuerdo;

2.-En desacuerdo;

3.-Neutral;

4.-De acuerdo; y

5.-Totalmente de acuerdo. 